\title{
The magnetic field around late-type stars revealed by the circumstellar $\mathrm{H}_{2} \mathrm{O}$ masers
}

\author{
W. H. T. Vlemmings ${ }^{1}$, H. J. van Langevelde ${ }^{2}$, and P. J. Diamond ${ }^{3}$ \\ ${ }^{1}$ Department of Astronomy, Cornell University, Ithaca, NY 14853, USA \\ e-mail:wouter@jb.man.ac.uk \\ 2 Joint Institute for VLBI in Europe, Postbus 2, 7990 AA Dwingeloo, The Netherlands \\ 3 Jodrell Bank Observatory, University of Manchester, Macclesfield, Cheshire, SK11 9DL, UK
}

Received 6 December 2004 / Accepted 20 January 2005

\begin{abstract}
Through polarization observations, circumstellar masers are excellent probes of the magnetic field in the envelopes of late-type stars. Whereas observations of the polarization of the $\mathrm{SiO}$ masers close to the star and on the $\mathrm{OH}$ masers much further out were fairly commonplace, observations of the magnetic field strength in the intermediate density and temperature region where the $22 \mathrm{GHz} \mathrm{H} \mathrm{H}_{2} \mathrm{O}$ masers occur have only recently become possible. Here we present the analysis of the circular polarization, due to Zeeman splitting, of the $\mathrm{H}_{2} \mathrm{O}$ masers around the Mira variable stars U Her and U Ori and the supergiant VX Sgr. We present an upper limit of the field around U Her that is lower but consistent with previous measurements, reflecting possible changes in the circumstellar envelope. The field strengths around U Ori and VX Sgr are shown to be of the order of several Gauss. Moreover, we show for the first time that large scale magnetic fields permeate the circumstellar envelopes of an evolved star; the polarization of the $\mathrm{H}_{2} \mathrm{O}$ masers around VX Sgr reveals a dipole field structure. We discuss the coupling of the magnetic field with the stellar outflow, as such fields could possibly be the cause of distinctly aspherical mass-loss.
\end{abstract}

Key words. masers - polarization - stars: circumstellar matter - stars: magnetic fields - stars: AGB and post-AGB

\section{Introduction}

At the end of their evolution, a large majority of stars go through a period of high mass loss while climbing the asymptotic giant branch (AGB). This mass loss, of the order of $10^{-7}$ to $10^{-4} M_{\odot} \mathrm{yr}^{-1}$, is responsible for the formation of a circumstellar envelope (CSE). It is also the main source for replenishing interstellar space with processed material. AGB stars such as the Mira variable stars, with main sequence masses less than a few $M_{\odot}$, will eventually develop as planetary nebulae (PNe). The heavier evolved stars such as the supergiants will eventually explode as a supernova ( $\mathrm{SN})$. The exact role of magnetic fields in the mass loss mechanism and the formation of CSEs is still unclear but could be considerable. The study of several maser species found in CSEs has revealed important information about the strength and structure of magnetic fields throughout the envelopes surrounding the late-type stars. At distances from the central star of up to several thousands of $\mathrm{AU}$, measurements of the Zeeman effect on $\mathrm{OH}$ masers indicate magnetic fields strengths of a few milliGauss (e.g. Szymczak \& Cohen 1997; Masheder et al. 1999). Additionally, weak alignment with the CSE structure is found (e.g. Etoka \& Diamond 2004). Observations of $\mathrm{SiO}$ maser polarization have shown highly ordered magnetic fields close to the central star, at radii of 5-10 AU where the $\mathrm{SiO}$ maser emission occurs (e.g. Barvainis et al. 1987; Kemball \& Diamond 1997). When interpreting the circular polarization of the $\mathrm{SiO}$ masers as standard Zeeman splitting, the magnetic field strength determined from these observations could be up to several tens of Gauss. However, a non-Zeeman interpretation can explain the observations by magnetic field strengths of only several tens of milliGauss (Wiebe \& Watson 1998).

Recently, high circular polarization of circumstellar $\mathrm{H}_{2} \mathrm{O}$ masers was observed for a small sample of late-type stars (Vlemmings et al. 2002, hereafter V02). $\mathrm{H}_{2} \mathrm{O}$ masers occur at intermediate distances in the CSE, in gas that is a factor of 10-1000 more dense than the gas in which $\mathrm{OH}$ masers occur. Vlemmings et al. (2001, hereafter V01) managed to detect the Zeeman splitting of the circumstellar $\mathrm{H}_{2} \mathrm{O}$ maser around the supergiant star S Per, even though for typical field strengths of a few hundred $\mathrm{mG}$ the $\mathrm{H}_{2} \mathrm{O}$ Zeeman splitting is extremely small, only $\approx 10^{-3}$ times the typical half-power width of the $\mathrm{H}_{2} \mathrm{O}$ maser line $\left(\Delta v_{L} \approx 30 \mathrm{kHz}\right)$. Additional detections were discussed in V02 and typical field strength of between 0.2 and $1 \mathrm{G}$ were found, using the full, non-LTE radiative transfer equations from Nedoluha \& Watson (1992). Such high magnetic field strengths at a few hundred AU from the central star give strong support for the standard Zeeman interpretation of the $\mathrm{SiO}$ maser polarization.

The results from V02 indicate that the magnetic field strengths measured in the circumstellar maser regions are consistent with either a $r^{-2}$ field strength dependence on the increasing distance to the star, similar to a solar-type magnetic field or possibly an $r^{-3}$ dependence as produced by a dipole 
Table 1. Star sample.

\begin{tabular}{lccccccc}
\hline \hline Name & Type & $\begin{array}{c}\text { RA (J2000) } \\
(\mathrm{h} \mathrm{m} \mathrm{s})\end{array}$ & $\begin{array}{c}\text { Dec }(\mathrm{J} 2000) \\
\left({ }^{\circ}, "\right)\end{array}$ & $\begin{array}{c}\text { Distance } \\
(\mathrm{pc})\end{array}$ & $\begin{array}{c}\text { Period } \\
(\text { days })\end{array}$ & $\begin{array}{c}V_{\text {rad }} \\
\left(\mathrm{km} \mathrm{s}^{-1}\right)\end{array}$ & $\begin{array}{c}\text { Peak flux } \\
(\mathrm{Jy})\end{array}$ \\
\hline VX Sgr & Supergiant & 180804.0485 & -221326.614 & $1700^{a}$ & 732 & 5.3 & 43.2 \\
U Her & Mira & 162547.4713 & +185332.867 & $277^{b}$ & 406 & -14.5 & 9.75 \\
U Ori & Mira & 055549.1689 & +201030.687 & $300^{c}$ & 368 & -38.1 & 6.44 \\
R Cas & Mira & 235824.8725 & +512319.703 & $176^{b}$ & 430 & 26.0 & - \\
\hline
\end{tabular}

${ }^{a}$ Marvel (1996); ${ }^{b}$ Vlemmings et al. (2003); ${ }^{c}$ Knapp et al. (1998).

magnetic field. This implies surface magnetic fields of hundreds to several thousand Gauss, indicating that the magnetic field pressure dominates the thermal and radiation pressure at the surface of the star. As a result, the magnetic field likely plays a very important role in driving the late-type star mass loss. Additionally, magnetic fields possibly play an important role in shaping the CSEs. This will also affect the formation of PNe, that are often observed to have distinctly non-spherical shapes.

Here we present new results on the magnetic field strength in the envelopes of several late-type stars. We discuss the observations and data calibration in Sect. 2 and the analysis method in Sect. 3. The new and previous results for our sources are presented in Sect. 4. In Sect. 5 the specific results on the supergiant VX Sgr are discussed, where the maser polarization observations allow for the investigation of the morphology of the magnetic field. The consequences of these measurements are discussed in Sect. 6 and are followed by our conclusions in Sect. 7.

\section{Observations}

The observations were performed at the NRAO Very Long Baseline Array (VLBA) on April 20 2003. The average beam width is $\approx 0.5 \times 0.5$ mas at the frequency of the $6_{16}-5_{23}$ rotational transition of $\mathrm{H}_{2} \mathrm{O}, 22.235 \mathrm{GHz}$. We used 4 baseband filters of $1 \mathrm{MHz}$ width, which were overlapped to get a velocity coverage of $\approx 44 \mathrm{~km} \mathrm{~s}^{-1}$, covering most of the velocity range of the $\mathrm{H}_{2} \mathrm{O}$ masers. Similar to the observations in V02, the data were correlated multiple times. The initial correlation was performed with modest $\left(7.8 \mathrm{kHz}=0.1 \mathrm{~km} \mathrm{~s}^{-1}\right)$ spectral resolution, which enabled us to generate all 4 polarization combinations (RR, LL, RL and LR). Two additional correlator runs were performed with high spectral resolution $\left(1.95 \mathrm{kHz}=0.027 \mathrm{~km} \mathrm{~s}^{-1}\right)$ which therefore only contained the two polarization combinations RR and LL. This was necessary to be able to detect the signature of the $\mathrm{H}_{2} \mathrm{O}$ Zeeman splitting in the circular polarization data and to cover the entire velocity range of the $\mathrm{H}_{2} \mathrm{O}$ masers. Each source-calibrator pair was observed for $6 \mathrm{~h}$. The calibrator was observed for $1.5 \mathrm{~h}$ in a number of scans equally distributed over the $6 \mathrm{~h}$.

\subsection{Calibration}

The data analysis path is described in detail in V02. It follows the method of Kemball et al. (1995) and was performed in the Astronomical Image Processing Software package (AIPS). The calibration steps were performed on the data-set with modest spectral resolution. We were also forced to flag several channels that suffered from strong interference. Fringe fitting and self-calibration were performed on a strong maser feature. The calibration solutions were then copied and applied to the high spectral resolution data-set. Finally, corrections were made for instrumental feed polarization using a range of frequency channels on the maser source, in which the expected frequency averaged linear polarization is close to zero. Then image cubes were created for stokes $I, Q, U$ and $V$ in the modest spectral resolution data set, and for stokes $I$ and $V$ in the high spectral resolution data-set.

\subsection{Sources}

We observed 4 late-type stars, the supergiant VX Sgr and the Mira variable stars U Ori, R Cas and U Her. U Her was previously observed in December 1998 as part of the observations described in V02. Unfortunately, we were unable to detect the $\mathrm{H}_{2} \mathrm{O}$ masers of $\mathrm{R}$ Cas, presumably due to source variability. In Table 1 we list the observed sources with type, position, distance, period and velocity, as well as the peak $\mathrm{H}_{2} \mathrm{O}$ maser flux at the epoch of observation. In the high spectral resolution total intensity channel maps, the noise is dominated by dynamic range effects and is $\approx 60 \mathrm{mJy}$. In the circular polarization polarization maps the rms noise is $\approx 20 \mathrm{mJy}$.

The polarization of the $\mathrm{OH}$ masers of our sources has previously been observed and $\mathrm{SiO}$ maser polarization measurements have been performed on VX Sgr. Polarization of the $1612 \mathrm{MHz} \mathrm{OH}$ masers around $\mathrm{U}$ Ori indicate a magnetic field of $B_{\mathrm{OH}} \approx 10 \mathrm{mG}$ (Reid et al. 1979), while Palen \& Fix (2000) found a field of $B_{\mathrm{OH}} \approx 1 \mathrm{mG}$ on the 1665 and $1667 \mathrm{MHz} \mathrm{OH}$ masers around $\mathrm{U}$ Her. The magnetic fields in the main-line and satellite-line $\mathrm{OH}$ maser regions around VX Sgr were measured using MERLIN (Chapman \& Cohen 1986; Trigilio et al. 1998; Szymczak et al. 2001) and the VLA (Zell \& Fix 1996). The polarization of the 1665 and $1667 \mathrm{MHz} \mathrm{OH}$ masers indicated a field strength of $B_{\mathrm{OH}} \approx 2 \mathrm{mG}$, while the field in the $1612 \mathrm{MHz}$ maser region is $B_{\mathrm{OH}} \approx 1 \mathrm{mG}$. In the single dish observations of the circular polarization of $\mathrm{SiO}$ masers by Barvainis et al. (1987), they observe a circular polarization percentage of $8.7 \%$. This indicates $B_{\mathrm{SiO}} \approx 90 \mathrm{G}$ for VX Sgr.

\section{Method}

For the analysis of the polarization spectra we used both the basic LTE interpretation and the full radiative transfer nonLTE interpretation, which were thoroughly described in V02. The LTE analysis consists of a standard Zeeman interpretation 
assuming LTE. As a result, the narrowing and rebroadening of the maser profile caused by saturation are not reproduced. We have included the possibility of multiple masing hyperfine lines. In this analysis, the magnetic field strengths are determined by fitting a synthetic $V$-spectrum, proportional to $\mathrm{d} I / \mathrm{d} v$, to the polarization spectra. The magnetic field strengths follow from

$$
\begin{aligned}
P_{V} & =\left(V_{\max }-V_{\min }\right) / I_{\max } \\
& =2 \cdot A_{\mathrm{F}-\mathrm{F}^{\prime}} \cdot B_{[\text {Gauss }]} \cos \theta / \Delta v_{\mathrm{L}}\left[\mathrm{km} \mathrm{s}^{-1}\right],
\end{aligned}
$$

with $P_{V}$ the percentage of circular polarization, $V_{\max }$ and $V_{\min }$ the maximum and minimum of the synthetic LTE $V$-spectrum fitted to the observations. $I_{\max }$ and $\Delta v_{\mathrm{L}}$ are the peak flux and the full width half-maximum $(F W H M)$ of the maser feature respectively. $B$ is the magnetic field strength at an angle $\theta$ from the line of sight. The $A_{\mathrm{F}-\mathrm{F}^{\prime}}$ coefficient depends on the masing hyperfine component and in V02 was estimated to be $\approx 0.011$, which is used here for all LTE estimates.

For the non-LTE analysis, the coupled equations of state for the 99 magnetic substates of the three dominant hyperfine components from Nedoluha \& Watson (1992) were solved for a linear maser in the presence of a magnetic field. The modeled spectra were then directly fitted to the observed spectra. When a direct model fit was not possible, we used Eq. (1) with $A_{\mathrm{F}-\mathrm{F}^{\prime}}=0.018$, which is the corresponding best estimate for the coefficient in the non-LTE case.

The spectral fitting for both the non-LTE as well as the LTE analysis requires the removal of the scaled down total power spectrum from the $V$-spectrum to correct for small residual gain errors between the right- and left-polarized antenna feeds. It was found in V01 and in V02 that generally, the $V$-spectra were narrower than expected in the LTE case. This could be partly explained by introducing the non-LTE method, however, some narrowing remained. In V02, we attributed this to the application of uni-directional maser propagation in the modeling, and we found that a bi-directional maser could explain some of the narrowing. However, for computational efficiency we continue to use the uni-directional method, while allowing for additional narrowing of the $V$-spectrum, as we found that the estimates for the fractional circular polarization are robust.

\section{Results}

We have determined the magnetic field strength on the strongest maser features in the band $\left(44 \mathrm{~km} \mathrm{~s}^{-1}\right.$ wide). The results of both the LTE and non-LTE analysis are shown in Table 2 for features with peak fluxes down to $\sim 5 \%$ of the brightest maser spot. As seen in Col. 6, the percentage circular polarization is relatively large and varies between $0.3 \%$ and $20 \%$ with the highest polarization being detected for U Ori. Magnetic field strengths obtained using the LTE Zeeman method are given in Col. 7 while the results obtained using the non-LTE models are shown in Col. 8. Features that did not show circular polarization at a level higher than 3 times the rms noise in the $V$-spectrum are considered non-detections. However, the formal errors on the magnetic field strength also include the errors on total intensity and line width, and are

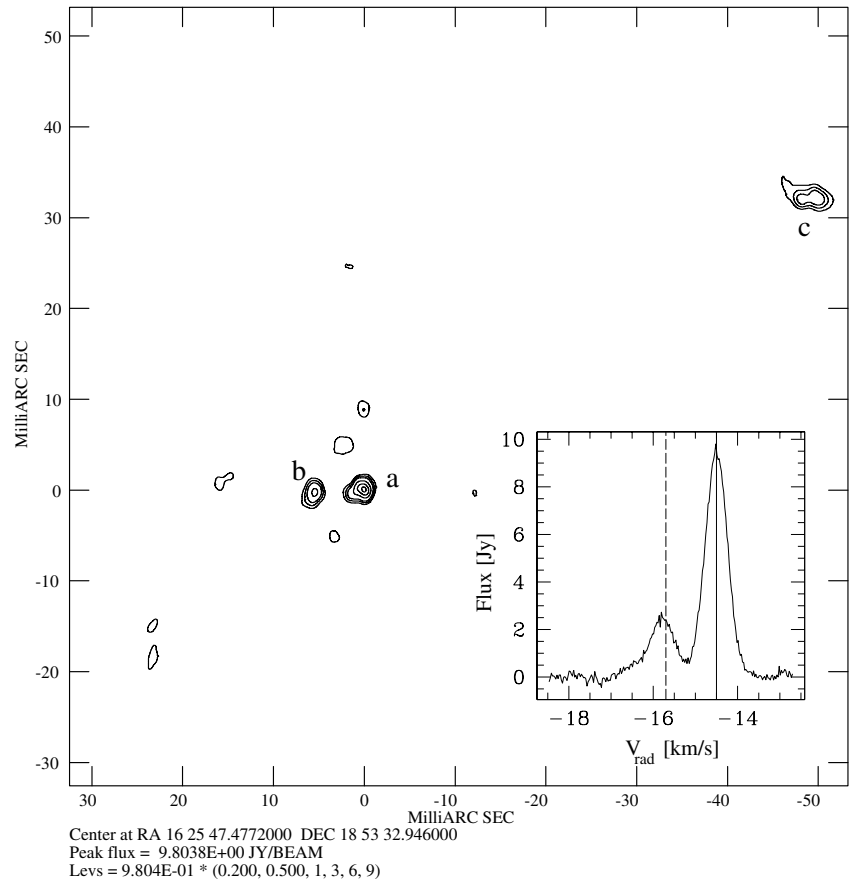

Fig. 1. Total intensity image of the $\mathrm{H}_{2} \mathrm{O}$ maser features around $\mathrm{U}$ Her. The inlay shows the total intensity spectrum of the $\mathrm{U}$ Her $\mathrm{H}_{2} \mathrm{O}$ masers, where the solid vertical line indicates the stellar velocity and the dashed vertical line indicates the velocity of the maser feature that was found to be coincident with stellar position in Vlemmings et al. (2002).

thus larger, resulting in occasional magnetic field strengths around the $1 \sigma$ noise level. For the non-detections we have determined the absolute upper limits using the $A_{\mathrm{F}-\mathrm{F}^{\prime}}$ coefficient obtained in the non-LTE radiative transfer models. In the LTE analysis the limits would be larger by a factor of $\approx 1.4$

Maser features that had circular polarization spectra that could not be fitted accurately are labeled in Table. 2. This is likely due to blending of several features, both spatially and along the velocity axis. The values for the magnetic field strength for those features were determined using only the minimum $V_{\min }$ and maximum $V_{\max }$ of the $V$-spectrum after it was forced to be point symmetric. The features labeled $* *$ (VX Sgr n, shown in Fig. 4 and VX Sgr w) suffered from external interference, most of which we managed to remove. Some effects remain however, and it is unclear if this had an effect on the measured magnetic field strength for those features.

As in V02, we were unable to detect any significant linear polarization above a limit of $\sim 0.02 \%$ for the strongest maser features and a few $\%$ for most of the weakest. There was a hint of linear polarization on the strongest maser feature of U Ori, but as the level of polarization was at most only at the $1 \sigma$ level of $\approx 2 \%$ it cannot unambigiously be classified as a detection.

\section{1. $U$ Her}

After the observations of $\mathrm{U}$ Her in $\mathrm{V} 02$ revealed a magnetic field in the $\mathrm{H}_{2} \mathrm{O}$ maser region of $\sim 1.5 \mathrm{G}$, U Her was observed again in our second observational run. The integrated total intensity map of the $\mathrm{H}_{2} \mathrm{O}$ maser features is shown in Fig. 1. Even though the strength of the U Her maser features is 
Table 2. Results.

\begin{tabular}{|c|c|c|c|c|c|c|c|}
\hline Name & Feature & $\begin{array}{c}\begin{array}{c}\text { Flux (I) } \\
(\mathrm{Jy})\end{array} \\
\end{array}$ & $\begin{array}{c}V_{\mathrm{rad}} \\
\left(\mathrm{km} \mathrm{s}^{-1}\right)\end{array}$ & $\begin{array}{c}\Delta v_{\mathrm{L}} \\
\left(\mathrm{km} \mathrm{s}^{-1}\right)\end{array}$ & $\begin{array}{c}P_{V} \\
\left(\times 10^{-3}\right)\end{array}$ & $\begin{array}{c}B \cos \theta \text { LTE } \\
(\mathrm{mG})\end{array}$ & $\begin{array}{c}B \cos \theta \text { non-LTE } \\
(\mathrm{mG})\end{array}$ \\
\hline \multirow[t]{25}{*}{ VX Sgr } & $\mathrm{a}$ & 18.8 & -2.01 & 0.47 & 21.0 & $350 \pm 72$ & $512 \pm 90$ \\
\hline & $b^{*}$ & 11.7 & -1.28 & 0.38 & 22.6 & $-307 \pm 99$ & $-248 \pm 62$ \\
\hline & $\mathrm{c}$ & 6.9 & 7.18 & 0.61 & 171.2 & $3705 \pm 249$ & $3375 \pm 265$ \\
\hline & $\mathrm{d}$ & 23.1 & 7.16 & 0.52 & 32.5 & $-609 \pm 67$ & $-469 \pm 60$ \\
\hline & $\mathrm{e}$ & 18.9 & 6.81 & 0.53 & 19.2 & $359 \pm 79$ & $249 \pm 46$ \\
\hline & $\mathrm{f}$ & 36.7 & 16.43 & 0.57 & & & $<54$ \\
\hline & $\mathrm{g}$ & 43.2 & 13.77 & 0.58 & 3.5 & $-75 \pm 34$ & $-63 \pm 20$ \\
\hline & $\mathrm{h}$ & 9.6 & 11.86 & 0.57 & 15.5 & $-326 \pm 184$ & $-290 \pm 115$ \\
\hline & $\mathrm{i}$ & 23.3 & 12.73 & 0.48 & & & $<99$ \\
\hline & $\mathrm{j}$ & 9.4 & -0.9 & 0.62 & 16.4 & $-370 \pm 202$ & $-325 \pm 126$ \\
\hline & $\mathrm{k}$ & 6.3 & -0.7 & 0.56 & 14.4 & $285 \pm 281$ & $277 \pm 195$ \\
\hline & 1 & 14.9 & 6.73 & 0.51 & 66.7 & $1231 \pm 123$ & $966 \pm 120$ \\
\hline & $\mathrm{m}$ & 9.7 & 20.6 & 0.52 & 9.9 & $-188 \pm 140$ & $-167 \pm 87$ \\
\hline & $\mathrm{n}^{* *}$ & 14.5 & -0.1 & 0.87 & 162.7 & $-4954 \pm 238$ & $-4082 \pm 305$ \\
\hline & $\mathrm{o}$ & 6.8 & 21.19 & 0.71 & & & $<381$ \\
\hline & $\mathrm{p}$ & 6.3 & 22.17 & 0.62 & & & $<471$ \\
\hline & $q$ & 8.5 & 20.97 & 0.49 & & & $<239$ \\
\hline & $\mathrm{r}$ & 3.4 & 20.93 & 0.62 & & & $<687$ \\
\hline & s & 2.7 & 9.95 & 0.54 & & & $<987$ \\
\hline & $\mathrm{t}$ & 8.4 & 16.26 & 0.48 & & & $<217$ \\
\hline & $\mathrm{u}$ & 9.9 & 13.64 & 0.58 & 4.5 & $-94 \pm 154$ & $-85 \pm 99$ \\
\hline & $\mathrm{v}$ & 7.2 & 7.1 & 0.51 & 39.9 & $-738 \pm 194$ & $-366 \pm 75$ \\
\hline & $\mathrm{w}^{* *}$ & 4.7 & 1.17 & 0.56 & 109.6 & $2160 \pm 387$ & $2195 \pm 251$ \\
\hline & $\mathrm{x}$ & 5.1 & 18.24 & 0.69 & & & $<512$ \\
\hline & $\mathrm{y}$ & 6.8 & 2.52 & 0.46 & 61.1 & $1012 \pm 281$ & $921 \pm 208$ \\
\hline \multirow[t]{3}{*}{ U Her } & $a$ & 9.75 & -14.56 & 0.55 & & & $<127$ \\
\hline & $\mathrm{b}$ & 3.75 & -14.42 & 0.58 & & & $<418$ \\
\hline & $\mathrm{c}$ & 2.09 & -15.82 & 0.55 & & & $<610$ \\
\hline \multirow[t]{3}{*}{ U Ori } & $\mathrm{a}^{*}$ & 6.44 & -41.05 & 0.61 & 22.6 & $-517 \pm 152$ & $-401 \pm 96$ \\
\hline & $\mathrm{b}$ & 1.87 & -41.23 & 0.75 & 217.9 & $-5770 \pm 718$ & $-3192 \pm 425$ \\
\hline & $\mathrm{c}$ & 0.95 & -40.85 & 0.66 & 140.4 & $3462 \pm 1210$ & $1398 \pm 382$ \\
\hline
\end{tabular}

${ }^{*}$ No accurate fit possible (see text).

${ }^{* *}$ Suffer from interference (see text).

similar in both observational epochs, we did not detect any significant circular polarization in the observations presented here. The upper limit we find is $\sim 130 \mathrm{mG}$ on the strongest and $\sim 600 \mathrm{mG}$ on the weakest maser feature. However, the spectrum and spatial distribution of the masers has changed significantly in the more than 4 years between the observational epoch of December 13th 1998 and April 20th 2003. The velocity of the maser features observed in V02 (between -19.3 and $-17.6 \mathrm{~km} \mathrm{~s}^{-1}$ ) was several $\mathrm{km} \mathrm{s}^{-1}$ more blue-shifted than the maser features observed here, that are close to the stellar radial velocity of $-14.5 \mathrm{~km} \mathrm{~s}^{-1}$. Whereas the spatial distribution of the maser spots in V02 showed an elongated structure in the N-S direction, Fig. 1 indicates a significantly different structure. The weakest VLBI feature detected at $-15.82 \mathrm{~km} \mathrm{~s}^{-1}$ (labeled $c$ in Fig. 1) corresponds in velocity to the feature that was found to be the strongest in the MERLIN observations of Vlemmings et al. (2002). In those observations, this feature was found to be aligned with the optical position to within 15 mas and interpreted as the amplified stellar image. Assuming that the current feature at $-15.82 \mathrm{~km} \mathrm{~s}^{-1}$ is also the amplified stellar image we obtain a lower limit on the distance on the sky between the brighter $\mathrm{H}_{2} \mathrm{O}$ maser features and the star. For a distance to U Her of $277 \mathrm{pc}$, the projected separation between the star and the two brightest maser features is $\approx 16$ AU. Since the velocity of these maser features is close to the stellar velocity, we expect the true separation to be similar. Assuming that the maximum magnetic field strength in $\mathrm{V} 02$ was measured at the inner edge of the $\mathrm{H}_{2} \mathrm{O}$ maser region, which was found to be $\approx 12.5$ AU (Bains et al. 2003), the expected magnetic field strength at $16 \mathrm{AU}$ is $\approx 700 \mathrm{mG}$ assuming a dipole magnetic field configuration. The difference between the expected field strength and the measured upper limits could be due to a large angle $\theta>75^{\circ}$ between the magnetic field lines and the line of sight. Alternatively, some of the magnetic field strength difference could be due to different maser clump density by up to a factor of $\sim 10$, if the magnetic field lines are frozen into the dense clumps. We could also be observing actual variability in the magnetic field.

\subsection{U Ori}

Figure 2 shows the integrated total intensity $\mathrm{H}_{2} \mathrm{O}$ maser map of $\mathrm{U}$ Ori. Very little structure is observed and the maser 

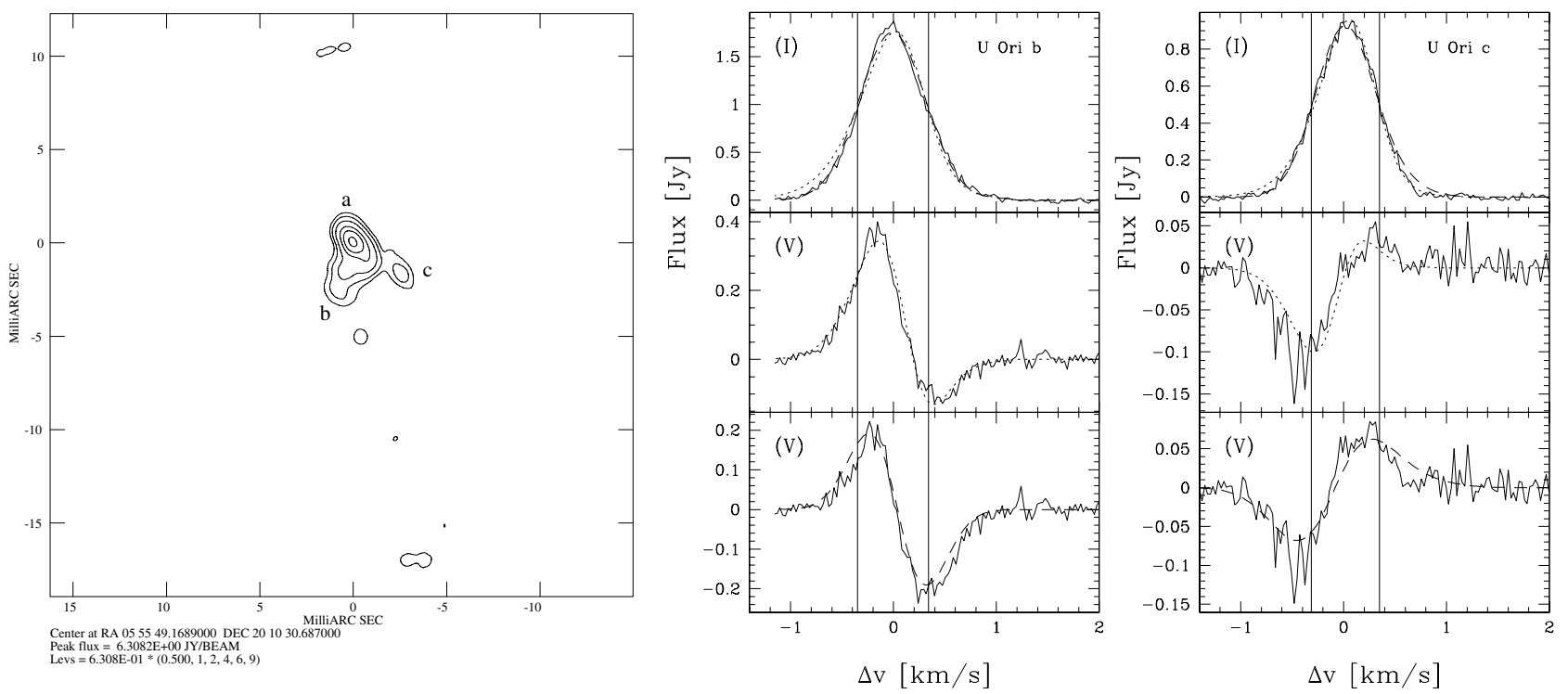

Fig. 2. (left) Total intensity image of the $\mathrm{H}_{2} \mathrm{O}$ maser features around $\mathrm{U}$ Ori. (right) Total power $(I)$ and $V$-spectra for selected maser features of U Ori. The bottom panel shows the best fitting synthetic $V$-spectrum produced by the standard LTE Zeeman interpretation (dashed line). The middle panel shows the best non-LTE model fit (dotted line). The corresponding total power fits are shown in the top panel. The $V$-spectra in the lower two panels are adjusted by removing a scaled down version of the total power spectrum as indicated in Sect. 3, which is different for the LTE and non-LTE fits. The solid vertical lines show the expected position of the minimum and maximum of the $V$-spectrum in the general LTE interpretation.

features seem to be somewhat blended both spatially as well as in velocity. Strong circular polarization up to $\approx 22 \%$ is detected on the three bright central features where interestingly the features $b$ and $c$, to the left and right of the brightest feature $a$, show an opposite sign for the magnetic field. The total intensity and circular polarization spectra for these features are also shown in Fig. 2. From these we estimate the absolute magnetic field strength in the maser region to be $\sim 3.5 \mathrm{G}$. While the central feature $a$ does not show such a high field strength, the feature seems to be a blend of two features with oppositely directed magnetic fields. The more complex circular polarization spectrum of U Ori $a$ can be explained by two features with a magnetic field of $\sim 800 \mathrm{mG}$ of opposite sign. The high magnetic field strength measured for the Mira variable star U Ori is consistent with the high field $(10 \mathrm{mG})$ measured using the $\mathrm{OH}$ masers (Reid et al. 1979) assuming a dipole $r^{-3}$ dependence of the magnetic field on the distance to the star. This assumes that the $\mathrm{H}_{2} \mathrm{O}$ masers occur in a shell with an outer edge of $30 \mathrm{AU}$, as determined by Bains et al. (2003).

\subsection{VX Sgr}

The integrated total intensity $\mathrm{H}_{2} \mathrm{O}$ maser map of the supergiant VX Sgr is shown in Fig. 3. VX Sgr shows a rich structure of strong $\mathrm{H}_{2} \mathrm{O}$ maser features in a $350 \times 250$ mas ellipse, corresponding to $600 \times 425 \mathrm{AU}$ at a distance of $1.7 \mathrm{kpc}$. The maser feature distribution is similar to that seen in the MERLIN observation by Murakawa et al. (2003, hereafter M03). Circular polarization was detected ranging from $0.3-16 \%$ for a large number of maser features as seen in Table 2. Several of the spectra are shown in Figs. 3 and 4. As mentioned above, a few of the maser features suffered from blending or external interference. The maximum magnetic field strength measured is $\sim 4 \mathrm{G}$. Across the maser features we find a clear transition between a negative magnetic field in the S-E to a positive magnetic field in the N-W. This is the first detection of a large scale magnetic field in a circumstellar $\mathrm{H}_{2} \mathrm{O}$ maser region.

\section{The morphology of the magnetic field around VX Sgr}

The maser shell of VX Sgr has been studied extensively with MERLIN and VLA observations (e.g. Lane 1984; Chapman \& Cohen 1986; Zell \& Fix 1996; Trigilio et al. 1998, M03). The recent observations of $\mathrm{M} 03$ indicate that the $\mathrm{H}_{2} \mathrm{O}$ masers arise in a thick shell between $100 \mathrm{AU}$ and $325 \mathrm{AU}$ from the star. In this shell, the $\mathrm{H}_{2} \mathrm{O}$ masers seem to be accelerated from $\sim 10 \mathrm{~km} \mathrm{~s}^{-1}$ to $20 \mathrm{~km} \mathrm{~s}^{-1}$. The $\mathrm{H}_{2} \mathrm{O}$ maser shell shows a clear elliptical asymmetry which was modeled in M03 as a spheroidal maser distribution intersected with an under-dense bi-conical region at an inclination angle $i=60^{\circ} \pm 30^{\circ}$ from the line of sight and rotated $\Theta=200^{\circ} \pm 30^{\circ}$ on the plane of the sky. Marvel (1996) also observed the elliptical $\mathrm{H}_{2} \mathrm{O}$ maser distribution with the VLBA and, constrained by proper motions, fitted the emission region using an oblate spheroid with a maximum radius of 250 mas at an inclination angle $i \sim 46^{\circ}$ and projected position angle $\Theta \sim 230^{\circ}$.

Observations of the $1612 \mathrm{MHz} \mathrm{OH}$ masers at $\sim 1400 \mathrm{AU}$ from the star also show the elliptical asymmetry observed in the $\mathrm{H}_{2} \mathrm{O}$ masers. The magnetic field strength in the $1612 \mathrm{MHz}$ $\mathrm{OH}$ maser region was estimated to be of the order of a milliGauss (Trigilio et al. 1998). Szymczak et al. (2001) observed the linear polarization of the $\mathrm{OH}$ masers and found structure that could be explained by a dipole magnetic field with an 

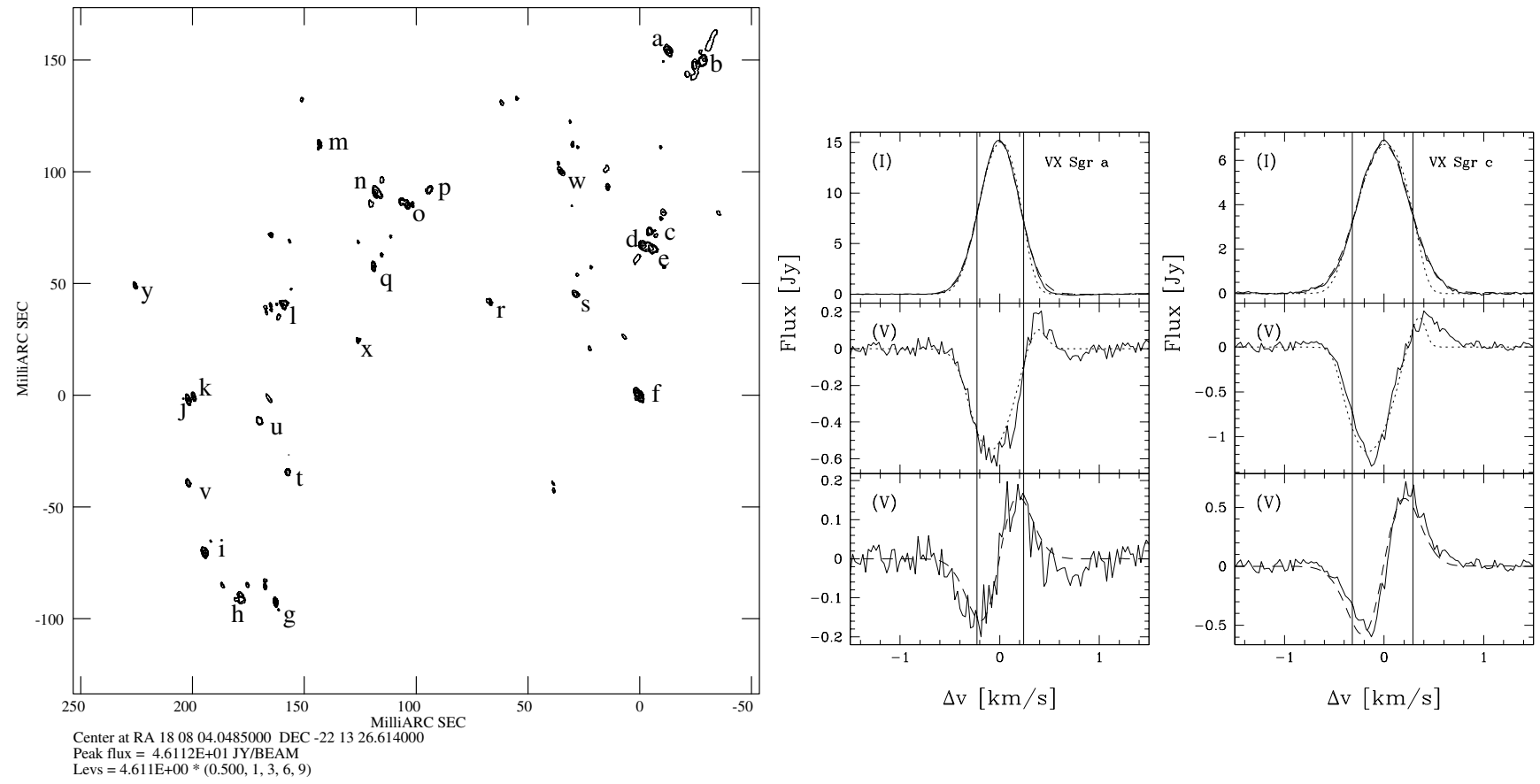

Fig. 3. Similar to Fig. 2 for VX Sgr.

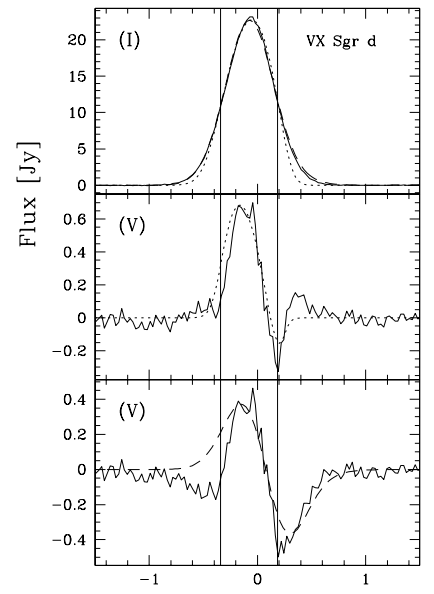

$\Delta \mathrm{v}[\mathrm{km} / \mathrm{s}]$

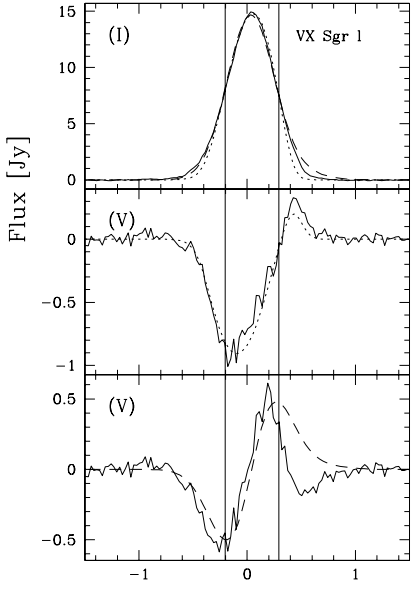

$\Delta \mathrm{v}[\mathrm{km} / \mathrm{s}]$

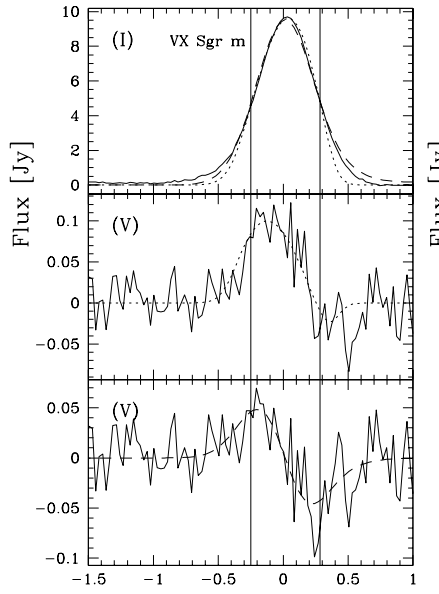

$\Delta \mathrm{v}[\mathrm{km} / \mathrm{s}]$

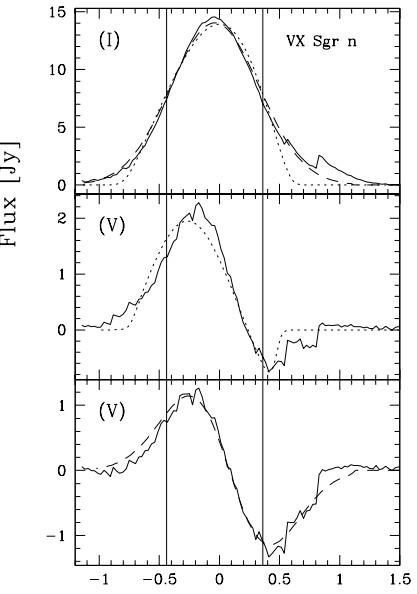

$\Delta \mathrm{v}[\mathrm{km} / \mathrm{s}]$

Fig. 4. Similar to the spectra in Figs. 2 and 3 for additional maser features around VX Sgr. Feature VX Sgr $n$ suffers from some interference effect in the right wing of the spectrum but this does not affect the magnetic field determination.

inclination axis of about $20^{\circ}$ or $30^{\circ}$. Additionally, the observations of Zeeman pairs in the $\mathrm{OH}$ emission indicates a change in magnetic field direction across the maser region. This was suggested to be the signature of a dipole magnetic field (Zell \& Fix 1996). Further observations indicated that the magnetic axis was projected at a position angle $\Theta=210^{\circ} \pm 20^{\circ}$ (Szymczak \& Cohen 1997). Observations of the $\mathrm{OH}$ mainline masers emission at $1667 \mathrm{MHz}$ closer to the star are consistent with the same magnetic angle (Richards et al. 2000).

We have fitted a dipole magnetic field to our $\mathrm{H}_{2} \mathrm{O}$ maser magnetic field observations of VX Sgr. As the positions of the maser features in the shell along the line of sight are unknown, we used the accelerating spherical outflow model of Chapman $\&$ Cohen (1986) to describe the velocity structure of the maser shell. Thus, the observed radial velocity of the maser features directly maps into the third spatial dimension, allowing for a three dimensional fit. A maximum likelihood fit was made to the dipole field, solving for the stellar position, the inclination and position angle of the magnetic field and the field strength at the surface of VX Sgr. The stellar radius was fixed at $\sim 16 \mathrm{AU}$, in agreement with the observations by Danchi et al. (1994) and Greenhill et al. (1995). In the fits we also included the maser features for which we only determined an absolute upper limit. The errors on the magnetic field strength were taken from our analysis, while we included errors of $1 \mathrm{~km} \mathrm{~s}^{-1}$ on the velocity of the maser features to take into account turbulent velocities and small deviations from the spherical outflow model. Figures 5 show the structure of the magnetic field as well as the 

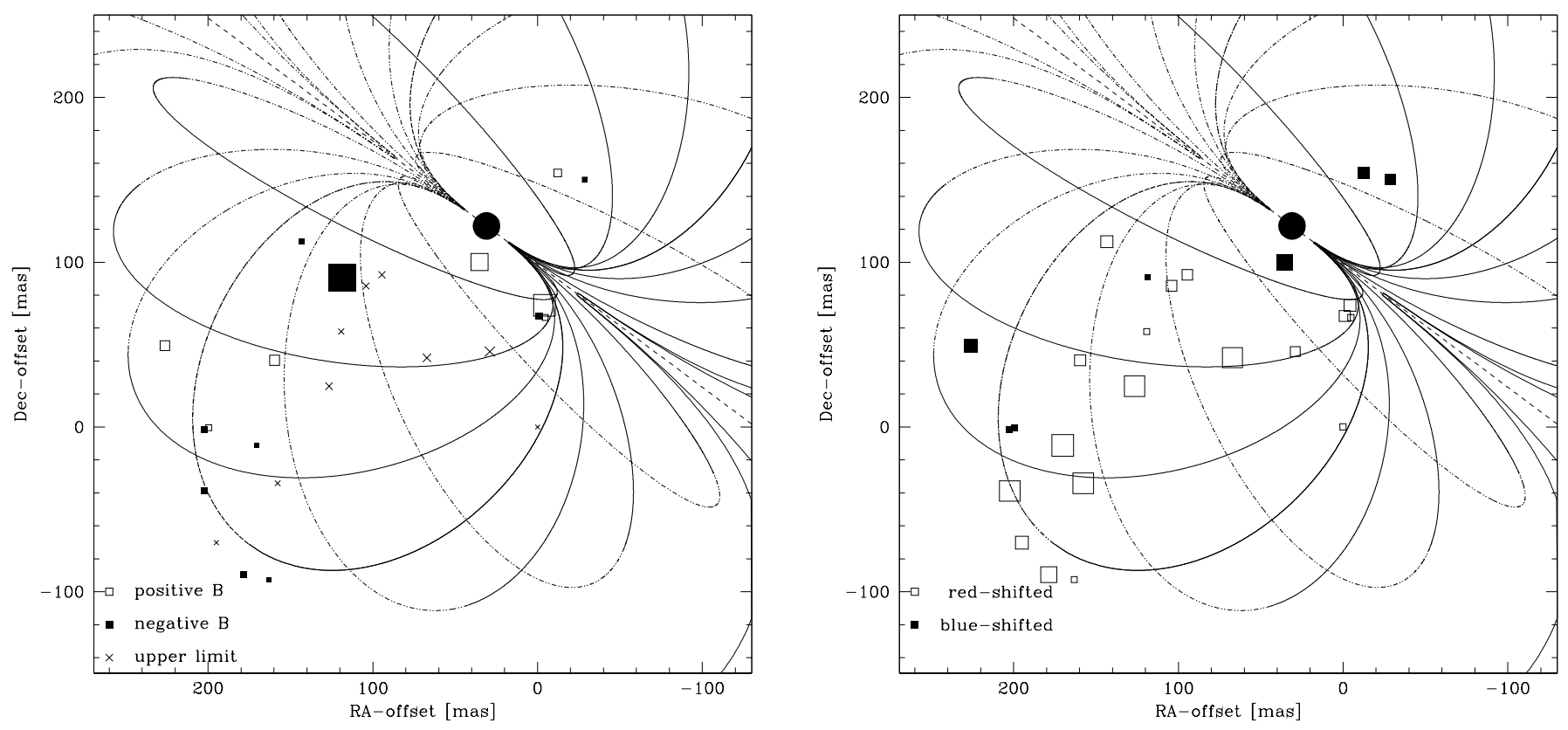

Fig. 5. The best fitted dipole magnetic field for the $\mathrm{H}_{2} \mathrm{O}$ maser observations around VX Sgr (denoted by the solid circle). (left) The distribution of the $\mathrm{H}_{2} \mathrm{O}$ maser features indicating the measured magnetic field strengths. Open symbols denote a positive magnetic field while the closed symbols correspond to a negative magnetic field. The crosses represent the upper limits. The symbols have been scaled relative to the magnetic field strength. (right) The distribution of the $\mathrm{H}_{2} \mathrm{O}$ maser features indicating the velocity structure of the maser features. The open symbols are the red-shifted features and the solid symbols are the blue-shifted features. The size indicates the velocity difference with the stellar velocity $\left(v_{\text {rad }}=5.3 \mathrm{~km} \mathrm{~s}^{-1}\right)$

radial velocity structure observed for the $\mathrm{H}_{2} \mathrm{O}$ maser features. The best fitted model for a dipole magnetic field is overplotted. While the magnetic field strengths can be fit reasonably well, the model has a harder time simultaneously fitting the velocity structure. Our fits show the stellar position to be offset from the center of the $\mathrm{H}_{2} \mathrm{O}$ maser emission by $\approx 70 \times 70$ mas to the NE. The magnetic axis of the dipole field is pointed toward us at an inclination angle $i=40 \pm 5^{\circ}$, and a position angle of $\Theta=220 \pm 10^{\circ}$. The indicated errors are the formal $1 \sigma$ errors from the fit. However, as both the magnetic field and the outflow velocity structure are likely to have small scale structure, the exact errors are strongly model dependent and are hard to quantify.

The fitted values are remarkably consistent with the values found for the magnetic field determined from $\mathrm{OH}$ masers, as well as with the orientation angle determined from the $\mathrm{H}_{2} \mathrm{O}$ maser distribution in M03 and Marvel (1996). We find that the magnetic field strength at the surface of VX Sgr corresponds to $B \approx 2.0 \pm 0.5 \mathrm{kG}$. For the $r^{-3}$ magnetic field strength dependence of the dipole field, this is consistent with the observations of the magnetic field on the $\mathrm{OH}$ masers as well as the $\mathrm{SiO}$ masers (Barvainis et al. 1987). In addition to a dipole magnetic field, we also considered a solar-type magnetic field with a magnetic field strength dependence of $r^{-2}$ on the distance to the star. This resulted in a significantly lower likelihood.

Our ability to perform such a fit of VX Sgr indicates that an ordered large scale magnetic field exists. Previously, an alternative model explained the magnetism in terms of local fields, frozen in high density pockets (Soker 2002). Although such small scale structure could still be important, we conclude that it is likely that large scale magnetic fields permeate the CSEs.

\section{Discussion}

\subsection{Circular polarization of $\mathrm{H}_{2} \mathrm{O}$ masers}

The observations reveal circular polarization percentages up to $\sim 20 \%$. Using the non-LTE radiative transfer method this indicates magnetic field strengths along the maser line of sight up to $\sim 4 \mathrm{G}$, while the fields determined using the LTE approximation are generally $\sim 40 \%$ higher. As shown in V02, the nonLTE circular polarization spectra are typically not symmetric, while the LTE method produces spectra with an point symmetric S-curve shape. However, due to the data processing and the necessary removal of a scaled down replica of the total power, it is impossible to directly observe the non-symmetric non-LTE spectra. Still, we confirm that the non-LTE spectra produce better fits to the observed $V$-spectra, as can be seen in several of the spectra presented in Figs. 2-4. Most of the observed $V$-spectra also show narrowing that cannot be reproduced in the LTE analysis, as was first found in V01. Similar to earlier results (e.g. Spencer et al. 1979; Vlemmings \& van Langevelde 2005), we find that the $\mathrm{H}_{2} \mathrm{O}$ masers are mostly unsaturated after an analysis of the line widths and shapes of the total intensity spectra.

We confirm in these observations again the absence of linear polarization to a limit of $\approx 2 \%$, which provides compelling evidence against the non-Zeeman interpretation, considered by Wiebe \& Watson (1998) for the SiO masers, as the cause for the observed high circular polarization of the $\mathrm{H}_{2} \mathrm{O}$ masers. 


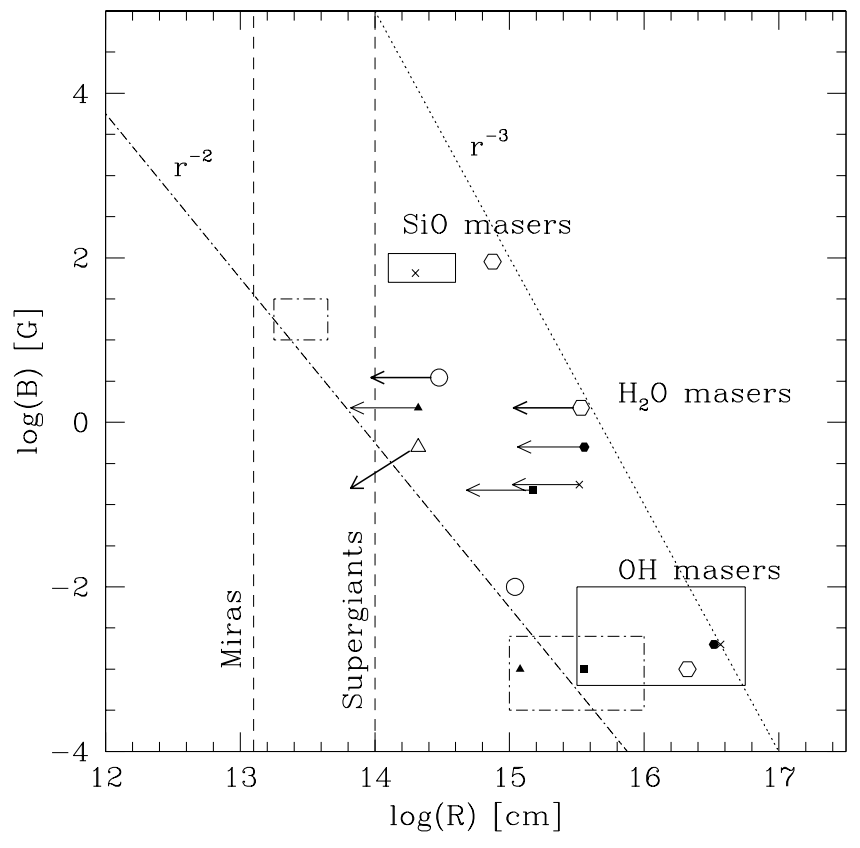

Fig. 6. Magnetic field strength, $B$, as function of distance, $R$, from the center of the star. Dashed-dotted boxes are the estimates for the magnetic fields in the $\mathrm{OH}$ and $\mathrm{SiO}$ maser regions of Mira stars, solid boxes are those for the supergiants. The dashed-dotted line indicates a solar-type magnetic field and the dotted line indicates the dipole field. The solid symbols are the previously observed stars (U Her: triangles; S Per: squares; VY CMa: crosses and NML Cyg: hexagonals). The open symbols indicate the stars of the sample presented here (U Her: triangle; VX Sgr: hexagonal and U Ori: circle). Note that the open triangle only indicates the upper limit determined for the most recent observation of U Her. Also note that the magnetic field strength observed on the $\mathrm{OH}$ masers of $\mathrm{U}$ Ori is larger than the typically observed fields for Mira variables. The dashed lines represent estimates of the stellar radius.

The fact that no significant linear polarization is observed is an additional indication that the circumstellar $\mathrm{H}_{2} \mathrm{O}$ masers are unsaturated (Nedoluha \& Watson 1991).

\subsection{The strength and shape of the magnetic field}

The observed magnetic field strengths on the $\mathrm{H}_{2} \mathrm{O}$ masers are consistent with the results for the other maser species assuming a $B \propto r^{\alpha}$ dependence of the field strength on the distance to the star. As the magnetic field strength determined for the $\mathrm{SiO}$, $\mathrm{H}_{2} \mathrm{O}$ and $\mathrm{OH}$ maser features depends on the angle between the magnetic field and the maser propagation axis, it is difficult to exactly determine the value of $\alpha$. In Fig. 6 we show an updated version of Fig. 15 from V02 including the results from the sources observed for this paper. The open symbols indicate the stars in the sample presented here. The points for the $\mathrm{H}_{2} \mathrm{O}$ masers are drawn at the outer radius of the maser region, which for VX Sgr was determined in M03. For U Ori we used the value of $\approx 30$ AU from Bains et al. (2003). The arrows indicate a typical width of the $\mathrm{H}_{2} \mathrm{O}$ maser region. The $\mathrm{SiO}$ maser location of $\approx 50 \mathrm{AU}$ for VX Sgr was taken from Doeleman et al. (1998) and its $\mathrm{OH}$ maser extent $(\approx 1400 \mathrm{AU})$ from Szymczak et al. (2001). The $\mathrm{OH}$ masers around U Ori were found to exist at $\approx 65$ AU (Chapman et al. 1991). For the other sources the references are listed in the relevant section of V02. We indicate a solar-type magnetic field $(\alpha=-2)$ and a dipole field $(\alpha=-3)$. The observations of VX Sgr indicate (as shown in Sect. 5) that a dipole field best fits the shape of the field in the $\mathrm{H}_{2} \mathrm{O}$ maser region. Extrapolating the observed magnetic field strengths to the stellar surface, we find that Mira variable stars have surface field strengths up to several times $10^{2} \mathrm{G}$, while supergiant stars have fields of the order of $10^{3} \mathrm{G}$.

\subsection{The origin of the magnetic field}

The origin of the strong, large scale magnetic fields around evolved stars remains a topic of debate. The generation of an axisymmetric magnetic field requires a magnetic dynamo in the interior of the star. One of the main arguments against a dynamo generated magnetic field is the slow rotation of the latetype star based on conservation of angular momentum (Soker \& Harpaz 1992). However, models by Blackman et al. (2001) consider the strong differential rotation created when the degenerated stellar core contracts while the envelope expands. In this case the dynamo is the result of the interaction between the differential rotation $(\omega)$ and turbulence in the convection zone around the degenerated core $(\alpha)$. This $\alpha \omega$-dynamo action can produce a strong magnetic field similar to the one observed using the circumstellar masers. It has been argued that such strong magnetic fields should produce strong X-ray emission. Although Mira is a weak X-ray source (Soker \& Kastner 2003), observations of TX Cam and T Cas did not reveal any X-ray emission (Kastner \& Soker 2004). However, as the optical depth for X-ray emission around evolved stars is expected to be high, it has been shown that this non-detection cannot rule out the strong magnetic fields produced by the $\alpha \omega$-dynamo (Blackman et al. 2001). The above models include the interaction with a degenerated core, while the core of supergiants such as VX Sgr is supposedly not degenerate. However, a similar dynamo action for supergiants, driven by the differential rotation between the contracting non-degenerate core and the expanding outer envelope has been shown to also be able to generate strong magnetic fields (Uchida \& Bappu 1982).

In contrast to the $\alpha \omega$-dynamo, several papers have examined a $\alpha^{2} \omega$-dynamo, under the assumption that the rotation of the envelope only marginally contributes to the dynamo action (Soker \& Zoabi 2002; Soker 2002). These models produce magnetic fields that are several orders of magnitude lower than the models using the $\alpha \omega$-dynamo. The observed magnetic field strength in the maser regions of the CSE are then argued to be due to localized, magnetized wind clumps that are produced in magnetic spots on the surface of the star that can have local field strengths of up to $10 \mathrm{G}$. Using another model, dynamo action of giant-cell convection at the surface of a late-type supergiant star has recently been shown to be able to produce fields up to $\sim 500 \mathrm{G}$ in localized spots on the stellar surface (Dorch 2004). Models that produce local strong field however, do not readily explain the observations of large scale magnetic field structure such as seen around VX Sgr. Alternatively, in the case of the $\alpha^{2} \omega$-dynamo a strong magnetic field can be 
generated when the star is spun up by a close binary. The sources in our sample do not however, show any indication of binarity, although this cannot be ruled out.

\subsection{Shaping of the circumstellar outflow}

The magnetic field strength of several Gauss measured in the $\mathrm{H}_{2} \mathrm{O}$ maser region implies that the magnetic pressure dominates the thermal pressure of the circumstellar gas throughout a large part of the CSE of both regular AGB stars and supergiants. The ratio between the thermal and magnetic pressure is given by $\left.\beta \equiv\left(8 \pi n_{\mathrm{H}} k T\right) / B^{2}\right)$, with $k$ the Boltzman constant. Assuming a gas density of $n_{\mathrm{H}}=10^{9} \mathrm{~cm}^{-3}$ and a temperature of $T \approx 10^{3} \mathrm{~K}$ at the inner edge of the $\mathrm{H}_{2} \mathrm{O}$ maser region, a magnetic field between $B=0.5$ and $1 \mathrm{G}$ gives $\beta \approx 0.012-0.003$, indicating that the magnetic pressure dominates the thermal pressure by factors of $\approx 80$ or more. In the lower density non-masing regions this factor will be even higher unless the magnetic field is frozen into the high density clumps. Still, in that case, when $B \propto n^{k}$ with $1 / 3 \leq k \leq 1 / 2$ (Mouschovias 1987), $\beta$ will be of the same order of magnitude.

The effects of magnetic fields on the stellar outflow and the shaping of the distinctly aspherical PNe have been discussed in several papers (e.g. Pascoli 1987; Chevalier \& Luo 1994; García-Segura 1997). PNe are thought to be formed due to the interaction of the slow AGB wind $\left(v \sim 10 \mathrm{~km} \mathrm{~s}^{-1}\right)$ with a subsequent fast wind $\left(v \sim 1000 \mathrm{~km} \mathrm{~s}^{-1}\right)$ generated when the central star evolves into a white dwarf (Kwok et al. 1978). Strong, large scale, magnetic fields around AGB stars could directly affect the fast wind and as a result shape the PN (Chevalier \& Luo 1994; García-Segura et al. 2003). Only recently, magnetic field strengths of $1-3 \mathrm{kG}$ have been detected at the surface of the central star of several PNe (Jordan et al. 2005). Another mechanism in which magnetism can play a role in shaping $\mathrm{PNe}$ is described in Matt et al. (2000). In the models presented in that paper, a large scale dipole magnetic field is responsible for creating an equatorial density enhancement in the initial, slow, AGB wind. For the magnetic field to influence the stellar outflow, the outflow must be sufficiently ionized, which is likely the case close to the surface of the star. A dipole magnetic field will then only need to have sufficient magnetic pressure $(\beta<2.0)$ to produce an equatorial disk. The interaction of the later fast wind with this disk has been shown to be able to easily create aspherical, cylindrical symmetric PNe (e.g. Icke 1988; Soker \& Livio 1989; Mellema et al. 1991). Furthermore, under the influence of the large scale magnetic field, the circumstellar disk could become warped (Lai 2003), and the interaction of the fast wind with such a disk has been shown by Icke (2003) to be able to explain the multipolar shape observed for several PNe, such as NGC 7027 (Cox et al. 2002). Although we have not directly observed the morphology of the magnetic field for the AGB stars in our sample, the magnetic field strengths measured on U Ori, are fully consistent with a dipole magnetic field such as found around the supergiant VX Sgr. As seen in Fig.3, we observe indeed that the $\mathrm{H}_{2} \mathrm{O}$ masers around VX Sgr occur in a oblate spheroid, which could be an indication of the equatorial density enhancement expected in a CSE that has been shaped by a dipole magnetic field.

\section{Conclusions}

We have measured the magnetic field around the late-type stars U Her, U Ori and VX Sgr using observations of the circular polarization of their $\mathrm{H}_{2} \mathrm{O}$ masers. Although we only find an upper limit in the case of U Her, we find strong magnetic fields of $\sim 3.5$ and $4 \mathrm{G}$ for U Ori and VX Sgr respectively. The rich structure of the $\mathrm{H}_{2} \mathrm{O}$ masers around the supergiant VX Sgr enabled us to determine the shape of the magnetic field around this supergiant star. The observations are best represented by a dipole magnetic field at angles that are remarkably consistent with those of the dipole field used to explain previous $\mathrm{OH}$ maser polarization observations at much larger distances from the central star. This confirms the presence of an ordered magnetic field close to the star. Additionally, the ellipsoidal structure of the $\mathrm{H}_{2} \mathrm{O}$ masers around VX Sgr is aligned with the equatorial plane of the dipole field, which could indicate the equatorial density enhancement caused by the magnetic field as described by Matt et al. (2000). The magnetic field strengths determined in the $\mathrm{H}_{2} \mathrm{O}$ maser regions around $\mathrm{U}$ Ori and $\mathrm{U}$ Her, lower mass evolved stars that are the progenitors of PNe, are also consistent with a dipole field, such as found around VX Sgr. As a result, also for those stars, the magnetic fields can cause aspherical density structures that result in non spherically symmetric PNe.

Acknowledgements. W.V. acknowledges the hospitality of the Harvard-Smithsonian CfA during his visit which was supported by the Niels Stensen Foundation.

\section{References}

Bains, I., Cohen, R. J., Louridas, et al. 2003, MNRAS, 342, 8

Barvainis, R., McIntosh, G., \& Predmore, C. R. 1987, Nature, 329, 613

Blackman, E. G., Frank, A., Markiel, J. A., Thomas, J. H., \& Van Horn, H. M. 2001, Nature, 409, 485

Chapman, J. M., \& Cohen, R. J. 1986, MNRAS, 220, 513

Chapman, J. M., Cohen, R. J., \& Saikia, D. J. 1991, MNRAS, 249, 227

Chevalier, R. A., \& Luo, D. 1994, ApJ, 421, 225

Cox, P., Huggins, P. J., Maillard, J.-P., et al. 2002, A\&A, 384, 603

Danchi, W. C., Bester, M., Degiacomi, C. G., Greenhill, L. J., \& Townes, C. H. 1994, AJ, 107, 1469

Doeleman, S. S., Lonsdale, C. J., \& Greenhill, L. J. 1998, ApJ, 494, 400

Dorch, S. B. F. 2004, A\&A, 423, 1101

Etoka, S., \& Diamond, P. 2004, MNRAS, 348, 34

García-Segura, G. 1997, ApJ, 489, L189

García-Segura, G., López, J. A., \& Franco, J. 2003, Rev. Mex. Astron. Astrofis. Conf. Ser., 15, 12

Greenhill, L. J., Colomer, F., Moran, J. M., et al. 1995, ApJ, 449, 365

Icke, V. 1988, A\&A, 202, 177

Icke, V. 2003, A\&A, 405, L11

Kastner, J. H., \& Soker, N. 2004, ApJ, 608, 978

Kemball, A. J., Diamond, P. J., \& Cotton, W. D. 1995, A\&AS, 110, 383

Kemball, A. J., \& Diamond, P. J. 1997, ApJ, 481, L111 
Knapp, G. R., Young, K., Lee, E., \& Jorissen, A. 1998, ApJS, 117, 209

Kwok, S., Purton, C. R., \& Fitzgerald, P. M. 1978, ApJ, 219, L125

Lane, A. P. 1984, VLBI and Compact Radio Sources, IAU Symp., 110, 329

Lai, D. 2003, ApJ, 591, L119

Masheder, M. R. W., van Langevelde, H. J., Richards, A. M. S., Greenhill, L., \& Gray, M. D. 1999, New Astron. Rev., 43, 563

Matt, S., Balick, B., Winglee, R., \& Goodson, A. 2000, ApJ, 545, 965

Marvel, K. B. 1996, Ph.D. Thesis, New Mexico State Univ.

Mellema, G., Eulderink, F., \& Icke, V. 1991, A\&A, 252, 718

Mouschovias, T. C. 1987, NATO ASIC Proc. 210: Physical Processes in Interstellar Clouds, 453

Murakawa, K., Yates, J. A., Richards, A. M. S., \& Cohen, R. J. 2003, MNRAS, 344, 1 (M03)

Nedoluha, G. E., \& Watson, W. D. 1991, ApJ, 367, L63

Nedoluha, G. E., \& Watson, W. D. 1992, ApJ, 384, 185

Palen, S., \& Fix, J. D. 2000, ApJ, 531, 391

Pascoli, G. 1987, A\&A, 180, 191

Reid, M. J., Moran, J. M., Leach, R. W., et al. 1979, ApJ, 227, L89

Richards, A. M. S., Cohen, R. J., Murakawa, K., et al. 2000, Proc. of the 5th EVN Symp., ed. J. E. Conway, A. G. Polatidis, R. S. Booth, \& Y. M. Pihlström, Publ. Onsala Space Observatory, 185

Soker, N. 2002, MNRAS, 336, 826
Soker, N., \& Livio, M. 1989, ApJ, 339, 268

Soker, N., \& Harpaz, A. 1992, PASP, 104, 923

Soker, N., \& Zoabi, E. 2002, MNRAS, 329, 204

Soker, N., \& Kastner, J. H. 2003, ApJ, 592, 498

Spencer, J. H., Johnston, K. J., Moran, J. M., Reid, M. J., \& Walker, R. C. 1979, ApJ, 230, 449

Jordan, S., Werner, K., \& O’Toole, S. J. 2005, A\&A, accepted

Szymczak, M., \& Cohen, R. J. 1997, MNRAS, 288, 945

Szymczak, M., Cohen, R. J., \& Richards, A. M. S. 2001, A\&A, 371, 1012

Trigilio, C., Umana, G., \& Cohen, R. J. 1998, MNRAS, 297, 497

Uchida, Y., \& Bappu, M. K. V. 1982, JA\&A, 3, 277

Vlemmings, W., Diamond, P. J., \& van Langevelde, H. J. 2001, A\&A, 375, L1 (V01)

Vlemmings, W. H. T., van Langevelde, H. J., \& Diamond, P. J. 2002, A\&A, 393, L33

Vlemmings, W. H. T., Diamond, P. J., \& van Langevelde, H. J. 2002, A\&A, 394, 589 (V02)

Vlemmings, W. H. T., van Langevelde, H. J., Diamond, P. J., Habing, H. J., \& Schilizzi, R. T. 2003, A\&A, 407, 213

Vlemmings, W. H. T., \& van Langevelde, H. J., 2005, 434, 1021

Wiebe, D. S., \& Watson, W. D. 1998, ApJ, 503, L71

Zell, P. J., \& Fix, J. D. 1996, AJ, 112, 252 